\title{
An Overview of Anesthesia Practices for Heart Transplant in India
}

\section{Sandeep Kumar Kar* and Pallav Mishra}

Assistant Professor, Cardiac Anesthesiology, IPGMER, Kolkata, India

*Corresponding Author: Sandeep Kumar Kar, Assistant Professor, Cardiac Anesthesiology, IPGMER, Kolkata, India.

Received: November 20, 2019

DOI: 10.31080/ASPS.2019.03.0449

\begin{abstract}
Heart transplant has seen a significant progress the number of patients with heart failure qualifying for cardiac transplantation is growing. Immunosuppressive agents have dramatically improved success of heart transplantation. Non-ischemic cardiomyopathy has surpassed ischemic cardiomyopathy as need for transplantation. The use of bridge therapy with mechanical circulatory support has improved both short-term and long-term survival of heart transplant recipients has gradually improved over time Keywords: Heart Transplant; Non-Ischemic Cardiomyopathy; Mechanical Circulatory Assist
\end{abstract}

\section{Introduction}

Organ Transplantation in India under aegis of National Organization Tissue Transplantation Organization (NOTTO) setup under Directorate General of Health Services established to oversee all donation and transplantation activities. In this regard, the entire country is having the following setup at National, Regional and State Level.

\begin{tabular}{|l|c|c|c|c|c|}
\hline $\begin{array}{c}\text { National } \\
\text { Level }\end{array}$ & \multicolumn{5}{|c|}{ (NOTTO) } \\
\hline $\begin{array}{c}\text { Regional } \\
\text { Level } \\
\text { (ROTTO) }\end{array}$ & $\begin{array}{c}\text { PGIMER } \\
\text { Chandigarh }\end{array}$ & $\begin{array}{c}\text { KEM } \\
\text { Hospital } \\
\text { Mumbai }\end{array}$ & $\begin{array}{c}\text { IPGME and } \\
\text { R Kolkata }\end{array}$ & $\begin{array}{c}\text { RGGGH } \\
\text { Chennai }\end{array}$ & $\begin{array}{c}\text { Guwahati } \\
\text { Medical } \\
\text { College }\end{array}$ \\
\hline $\begin{array}{l}\text { State } \\
\text { Level } \\
\text { (SOTTO) }\end{array}$ & & & & & \\
\hline
\end{tabular}

Table 1

The transplant registry for cardiac transplant is in the process of making data from various hospitals show greater number of heart transplant conducted in southern states of country showing Tamil Nadu with highest number of cardiac transplants undertaken.

History of heart transplant [1-4]

The International Society for Heart and Lung Transplantation (ISHLT) identifies 8 primary diagnoses for adult heart transplant recipients. The most common primary diagnosis has shifted over time from ischemic cardiomyopathy (ICM) to non-ICM (NICM), with NICM now representing $49.2 \%$ of all heart transplant recipi-

\begin{tabular}{|l|l|}
\hline $\begin{array}{l}\text { Dr. Christiaan } \\
\text { Neethling } \\
\text { Barnard }\end{array}$ & $\begin{array}{l}\text { st } \text { successful adult heart transplant on Mr. Louis } \\
\text { Washkansky 3 December 1967 at Groote Schuur } \\
\text { hospital Cape town, South Africa }\end{array}$ \\
\hline $\begin{array}{l}\text { Caves and } \\
\text { Colleagues }\end{array}$ & $\begin{array}{l}\text { Transvenous endomyocardial biopsy for diagnosis } \\
\text { of immune rejection of heart transplant in 1970. }\end{array}$ \\
\hline $\begin{array}{l}\text { Jean Francois } \\
\text { Borel }\end{array}$ & $\begin{array}{l}\text { Immunosuppressive effects of cyclosporin A as } \\
\text { preventive strategy to cardiac rejection in 1976. }\end{array}$ \\
\hline $\begin{array}{l}\text { Bruce Reitz } \\
\text { and Norman } \\
\text { Shumway }\end{array}$ & $1^{\text {st }}$ successful combined heart lung transplant [4]. \\
\hline
\end{tabular}

Table 2

ents. Ischemic cardiomyopathy remains the most prevalent diagnosis in patients older than 59 years. This trend continues even though older patients now represent an increasing proportion of the transplant population.

\begin{tabular}{|l|c|}
\hline \multicolumn{1}{|c|}{$\begin{array}{c}\text { Primary underlying diagnosis for } \\
\text { adult heart Transplant }\end{array}$} & $\begin{array}{c}\text { Frequency } \\
\mathbf{\%}\end{array}$ \\
\hline Non-Ischemic cardiomyopathy & 49.2 \\
\hline Ischemic cardiomyopathy & 34.6 \\
\hline Congenital heart disease & 3.2 \\
\hline Retransplantation & 3 \\
\hline Hypertrophic cardiomyopathy & 3 \\
\hline Valvular cardiomyopathy & 2.7 \\
\hline Other & 1.1 \\
\hline
\end{tabular}

Table 3 
Anaesthesia for heart transplant [5]

Heart transplant be considered for adults with the following criteria;

- $\quad$ Cardiogenic shock with low probability of recovery as associated with volume overload/inability to wean ventilation. Inability to wean temporary mechanical circulatory support such as IABP, VAD and ECMO supported patients. Inability to wean continuous inotropic support.

- $\quad$ NYHA Grade III-IV despite maximal medical and surgical therapy in conditions like hypertrophic/restrictive cardiomyopathy, complex congenital heart disease not amenable to surgical or procedural intervention.
- Severe functional limitation secondary to underlying cardiac condition with Peak oxygen consumption Peak V02 below $12-14 \mathrm{ml} / \mathrm{kg} / \mathrm{min}$ or serial decline over time in context to age, Six-minute walk test below 300 meters.

- Ischemic heart disease with Canadian Cardiac Society Class III/IV refractory angina pectoris despite optimal medical, surgical and interventional therapy.

- Recurrent life-threatening ventricular arrhythmia despite optimal medical, surgical and electrophysiological therapy.

- Localized cardiac tumor with low likelihood of metastasis.

\section{\begin{tabular}{|c|}
\hline Absolute Contraindication \\
\hline Systemic illness with a life expectancy $<2$ yrs
\end{tabular} despite heart transplant include the follow- ing;}

1. Active or recent solid organ or blood malignancy within 5 years.

2. AIDS with frequent opportunistic infection.

3. Systemic lupus erythematosus, sarcoid, or amyloidosis that has multisystem involvement and is still active.

4. Irreversible renal or hepatic dysfunction in patients considered for only heart transplantation.

5. Significant obstructive pulmonary disease $(\mathrm{FEV} 1<1 \mathrm{l} / \mathrm{min})$

6. Fixed pulmonary hypertension with Pulmonary artery systolic pressure $>60$ $\mathrm{mm} \mathrm{Hg}$. Mean transpulmonary gradient $>15 \mathrm{~mm}$ Hg. Pulmonary vascular resistance $>6$ wood units.

\section{Relative Contraindication}

Relative Contraindications;

1. Age $>72$ years

2. Any active infection (with exception of device-related infection in ventricular assist device recipients)

3. Active peptic ulcer disease.

4. Diabetes with end-organ damage

5. Severe peripheral vascular or cerebrovascular disease

6. Morbid obesity (BMI $>35 \mathrm{~kg} / \mathrm{m} 2)$ or cachexia $(\mathrm{BMI}<18 \mathrm{~kg} / \mathrm{m} 2)$

7. Serum creatinine $>2.5 \mathrm{mg} / \mathrm{dL}$ or creatinine clearance less than $25 \mathrm{ml} / \mathrm{min}$.

8. Bilirubin $>2.5 \mathrm{mg} / \mathrm{dL}$, serum transaminases $>3$ times normal, or INR $>1.5$ off warfarin.

9. Severe pulmonary dysfunction with FEV1 below 40\%.

10. Active mental illness or psychosocial instability 11. Drug, tobacco, or alcohol abuse within 6 months.

11. Recent pulmonary infarction within 6 to 8 weeks.

12. Irreversible neurologic or neuromuscular disorder.

Table 4

\section{Diseases specific candidate selection [5]}

Patient selection needs to be meticulously undertaken by identifying acute heart failure cases and managing critical issues prior to undertaking transplant procedure. This requires dedicated team work involving management of hemodynamics and intensifying medical management. Identifying acute heart failure.

\section{Diagnostic criteria}

NYHA class III-IV.

Heart failure decompensation characterized by volume overload/reduced cardiac output.
Severe cardiac dysfunction with LVEF below 30\% with pseudo normal/restrictive mitral inflow pattern.

\section{PCWP $>16$ or RAP $>12 \mathrm{mmHg}$}

BNP/NT Pro BNP rise in absence of noncardiac causes severely impaired functional capacity with inability to exercise, six-minute walk below 300 meter and Peak Vo2 less than 12-14ml/kg/min.

History of more than one heart failure hospitalization in past six months. 


\section{Clinical events suggesting acute Heart failure}

- Heart failure more than two hospitalization in emergency department in past 12 months.

- Progressive decline in renal function.

- $\quad$ Cardiac cachexia.

- Intolerance to ACE inhibitors due to hypotension or worsening renal failure.

- Intolerance to beta blocker due to hypotension.

- or worsening heart failure.

- Frequent Systolic blood pressure below 90mmHg.

- Persistent dyspnea with dressing/bathing requiring rest.

- Inability to walk one block on level ground because of dyspnea or fatigue.

- Escalation of diuretic to maintain euvolemic

- (furosemide more than $160 \mathrm{mg} /$ day or Metolazone use).

- Frequent ICD shock.

- Frequent hyponatremia below $133 \mathrm{meq} / \mathrm{ml}$.

\section{Anesthetic management of donor heart [9-12]}

Perioperative management of the heart transplant involves recipient-donor cross matching for $\mathrm{ABO}$ compatibility and donor's cardiac history with the degree of hemodynamic compromise Electrocardiography and echocardiography are mandatory. Coronary angiography is recommended for male donors over 45 years and females over 50 years. The heart retrieval team undertakes direct visualization before donor cardiectomy occurs. The donor heart size should be within $20 \%-30 \%$ of the recipient. Larger donor heart is chosen in patients with elevated pulmonary vascular resistance, the larger heart being better able to withstand the stress of higher pulmonary afterload. The anaesthetic management of the brain-dead donor is the maintenance of normovolaemia and normothermia to ensure organ perfusion. Avoidance of hypoxia using high inspired oxygen concentrations and Positive End Expiratory Pressure (PEEP) and maintaining adequate filling pressure. The oxygen carrying capacity of the blood is maximized using red cell transfusion if needed. Mean arterial pressure maintained around 80-90 mmHg with the help of vasopressor. The loss of central thermoregulatory mechanisms may lead to hypothermia with resultant arrhythmias. Ventilator management is critical as patients frequently develop neurogenic pulmonary edema or acute respiratory distress syndrome (ARDS). Central diabetes insipidus typically ensues leading to massive diuresis and electrolyte imbalances in brain dead patients.
Surgery involves a median sternotomy and pericardiotomy, systemic heparinization is accomplished and the superior vena cava is ligated. The aortic root is then cannulated for the administration of cardioplegia. After ligation of the great veins, the heart is exsanguinated and decompressed. Following the infusion of cardioplegia and cardiac arrest, the aorta is cross-clamped. Topical hypothermia by way of iced slush is sometimes applied to the heart. Donor cardiectomy is then performed leaving remnants of the posterior atrial walls, pulmonary veins and venae cava intact. Conventionally accepted donor heart ischemic time ranges from 3 to 6 hours. Cold ischemic time be minimized leading to innovations like the Trans Medics Portable Warm Blood Perfusion System (Organ Care System, Trans Medics Corporation, Andover, MA) which is currently under investigation in the United States. This system is designed to keep the donor heart warm and beating, maintaining pulsatile perfusion. Donor blood is used and the system provides blood oxygenation and flow from an internal gas supply and pulsatile pumping system. This has the advantage of enabling resuscitation of the donor organ, potentially improving its function and increasing the amount of time that an organ can be maintained outside the body in a condition suitable for transplantation by reducing time dependent ischemic injury. This system has been approved for use in the European Union since mid-2006.

\section{Anaesthetic concerns for cardiac transplant recipient [13-15]}

Heart transplant is considered an urgent or emergent surgery. Timing of transplantation include the time of donor surgery and organ transport, potential preoperative optimization of recipients, adequate time for induction of anesthesia and line placement, and appropriate recipient surgical timing to minimize ischemic time. Communication between the harvesting team, recipient surgical team and anesthesia team with each other to minimize delay at any stage be undertaken. The use of mechanical circulatory assist devices and prior knowledge is important as well as the antiarrhythmic devices their patency and sterility of monitoring and access lines be undertaken. Antiarrhythmic devices should be interrogated preoperatively and reprogrammed to a mode that will not misinterpret electrocautery interference.

Implantable cardioverter defibrillators should have the defibrillators inactivated.

Magnets should be available, and the effects of a magnet should be verified against each device. The antiarrhythmic devices in most cases are removed during the surgery after the donor heart is successfully transplanted. The preoperative evaluation should include 
an assessment of the patient's coagulation status and risk of bleeding. Laboratory studies should be reviewed; preoperative anemia, thrombocytopenia, and an elevated international normalized ratio are all associated with increased perioperative bleeding during cardiac surgery. Redo sternotomies, which are common given the increasing prevalence of ventricular assist devices, also increase the risk of perioperative bleeding. Blood availability should be there before incision. Large bore peripheral intravenous lines and a central venous catheter should be accessible in case rapid transfusion of blood products or fluids is required. There is significant variability in intraoperative blood product requirements during transplant. Multiple studies have shown that the duration of ischemic time has an adverse effect on survival. In an effort to minimize ischemia, the induction of anesthesia and recipient surgical incision should preferably begin as soon as the harvesting team has evaluated the donor heart and found it to be acceptable. Cardiopulmonary bypass should be initiated and the heart excised as soon as the donor heart arrives to the recipient hospital. The anesthesiologist should communicate his or her anticipated time for induction and line placement with the surgical teams. Clear, closed-loop communication with the transplant surgery team is imperative, because the timing of immunosuppressant medications is crucial. Coordination of care is key regarding timing for induction of anesthesia transplant recipient. Recent registry data demonstrated a statistically significant increase in acute graft failure in 1-year and 5-year mortality rates with ischemic times longer than 210 minutes. Minimize organ ischemic time, with a target goal of less than 4 hours. We must also account for the fact that these patients often have experienced multiple punctures of arteries and central veins. Additionally, given the emergency basis of these procedures, it is possible that patients may not be nil per oral and should be evaluated for the need of rapid sequence intubation.

Pre-operative workup shall include [18-20]

- Preanesthetic workup covering patient disease history, physical examination.

- Majority patients on vasopressors, inotropes or mechanical circulatory assist device be ascertained.

- Antiarrhythmic device use ascertained and programmed appropriately to avoid cautery interference.

- ICD should have the defibrillator inactivated and magnet available.

- Diabetes with end-organ damage (other than non-proliferative retinopathy) or persistent poor glycemic control (gly- cosylated hemoglobin [HbA1c] $47.5 \%$ or $58 \mathrm{mmol} / \mathrm{mol}$ ) despite optimal effort is a relative contraindication for transplant.

Renal function should be assessed using estimated glomerular filtration rate (eGFR) or creatinine clearance. Evidence of abnormal renal function should prompt further investigation, including renal ultrasonography, estimation of proteinuria and evaluation for renal arterial disease to exclude intrinsic renal disease. It is reasonable to consider the presence of irreversible renal dysfunction (eGFR of $30 \mathrm{ml} / \mathrm{min} / 1.73 \mathrm{~m} 2$ ) as a relative contraindication for heart transplantation alone.

Pre-transplant body mass index (BMI) $35 \mathrm{~kg} / \mathrm{m} 2$ is associated with a worse outcome after cardiac transplantation. For such obese patients, it is reasonable to recommend weight loss before listing for cardiac transplantation.

Clinically severe symptomatic cerebrovascular disease may be considered a contraindication to transplantation. Peripheral vascular disease may be considered a relative contraindication for transplantation when its presence limits rehabilitation and revascularization is not a viable option.

Tobacco use, substance abuse, and psychosocial evaluation in candidates.

Assessment of frailty ( 3 of 5 possible symptoms, including unintentional weight loss of 10 pounds within the past year, muscle loss, fatigue, slow walking speed, and low levels of physical activity) could be considered when assessing candidacy.

Psychosocial support as patient for whom social supports are deemed insufficient to achieve compliant care in the outpatient setting may be regarded as having a relative contraindication to transplant. The benefit of transplant in patients with severe cognitive-behavioral disabilities or dementia has not been established with the potential for harm so cardiac transplant cannot be recommended for this subgroup of patients.

- $\quad$ Latest pulmonary function test.

- $\quad$ Effort tolerance assessed by

- 6-minute walk test

- $\quad$ Dobutamine Stress Echo

- Transthoracic Echocardiography (2D)

- Right heart catheterization should be performed on all adult candidates in preparation for listing for cardiac transplantation and periodically until transplantation. 
- Coronary Angiography depending on Trans thoracic Echocardiography finding.

- Antibiotic selection requires evaluation of the donor and recipient's infection patterns and forthcoming immunosuppressants.

- Coagulation profile as many redo sternotomies can have significant bleeding in light of existing ventricular assist device.

\section{Disease specific consideration for cardiac transplant [5]}

Restrictive cardiomyopathy patients with severe heart failure symptoms (New York Heart Association Functional Classification III-IV) should be referred for transplant evaluation. The patients evaluated for transplant should undergo a complete diagnostic workup to elucidate etiology (infiltrative forms vs idiopathic) and to exclude constrictive pericarditis. The decision to list patient on the cardiac transplant waiting list should take into consideration specific prognostic indicators (the presence and degree of LV systolic dysfunction, atrial enlargement, pulmonary hypertension, and a low cardiac output. The efficacy and safety of LVAD as a bridge to transplant cannot be recommended as standard procedure. MCS with an LVAD or a total artificial heart may be considered in highly selected cases and at experienced centers (Class IIb, Level of Evidence: C).

Heart failure due to $\mathrm{AL}$ amyloidosis who are not candidates for disease-specific therapies due to cardiovascular compromise may be considered for transplant in experienced centers with established collaborations between cardiovascular and hematology teams. Autologous stem cells transplantation (ASCT) should be planned as soon as clinically feasible after recovery from cardiac transplant. Patients with transthyretin related (TTR) amyloidosis involving the heart may be considered for cardiac transplant. Familial TTR cardiac amyloidosis patients should be considered for combined heart and liver transplantation in experienced centers with established collaboration between cardiology, hepatology, and neurology teams.

Patients with severe heart failure and non-obstructive HCM not otherwise amenable to other treatment interventions should be considered for transplant. Particular attention should be given to those patients with LV dilation and systolic dysfunction.

Selected HIV-positive candidates may be considered for transplant if they have no active or prior opportunistic infections, are clinically stable and compliant on combination antiretroviral therapy (cART) for 43 months, have undetectable HIV RNA, and have
CD 4 counts 4200 cells/ $\mu$ l for 43 months Transplant centers performing heart transplantation in HIV-positive candidates should have structured protocols with multidisciplinary teams, adequate access to pharmacologic expertise, therapeutic drug monitoring for immunosuppressants, and laboratory access to antiviral drug resistance testing as needed. Candidates with a history of primary central nervous system lymphoma and visceral Kaposi sarcoma should not be considered for transplant. HIV-positive candidates with other resolved neoplasms, including squamous cell carcinoma of the skin, anogenital carcinoma in situ and other solid organ tumors considered cured may be considered after an appropriate disease-free period.

All transplant candidates should be screened for latent tuberculosis (TB) infection (LTBI) with a tuberculin skin test (TST) and/or interferon- $\gamma$ release assay (IGRA) where available a candidate has had a recent exposure to TB, or chest X-ray shows old TB (and inadequate or no treatment), 3 consecutive early morning sputum or bronchoalveolar lavage specimens should be obtained to exclude active TB disease Candidates with a positive IGRA or TST 5- mm induration should be treated pre-transplant with isoniazid, if tolerated. Candidates from a TB-endemic area with a positive IGRA or TST $5-\mathrm{mm}$ induration should have at least 1 other risk factor (evidence of a recent seroconversion, evidence of old TB lung disease, history of untreated or inadequately treated TB, close contact with a person with TB) before commencing isoniazid prophylaxis. Add pyridoxine (25-50 mg/day) during isoniazid therapy to avoid peripheral neurotoxicity. Treatment for LTBI should be for 6 to 9 months and should not interfere with the timing of transplantation. Patients should commence treatment as soon as possible before transplant and continue after transplant to compete a full course of therapy.

In candidates with resolved or prior inactive HCV infection, HCV RNA PCR testing should be performed at screening, at 3-month intervals while listed, and repeated at the time of transplantation. In candidates with resolved or prior inactive HBV infection, serology and DNA viral load testing should be performed at screening, at 3-month intervals while listed, and repeated at time of transplantation. Complete viral HBV evaluation before transplantation should also include $\mathrm{HBeAg}$ and $\mathrm{HBeAB}, \mathrm{HBcAB}$, immunoglobulin $\mathrm{G}$ and $\mathrm{M}$, and hepatitis delta virus (HDV) Ag, HDV AB, and serum $\alpha$-fetoprotein. In patients with chronic HCV infection, HCV genotype should be determined, and most patients will require a liver biopsy before active listing. In patients with chronic HBV infection, liver biopsy should be done in all patients to exclude severe dis- 
ease. In patients with chronic HCV or HBV infection, clinical, radiologic or biochemical signs of cirrhosis, portal hypertension, or hepatocellular carcinoma are contraindications to transplant.

Congenital heart disease transplant for CHD should only be performed at centers with established medical and surgical experience in both adult CHD and transplantation. All candidates with CHD should undergo detailed assessment of the position and anatomy of the abnormalities within the chest (via cardiac magnetic resonance imaging or chest computed tomography) to guide the surgical strategy, evaluation of PVR, and identification of all potential sources of pulmonary flow, assessment of patency of major veins and arteries and venous collaterals across the chest wall, presence of chronic or previous infections, presence of disease in organ systems that can affect post-transplant care and/ or cannot be reversed with transplantation, qualitative and quantitative assessment of anti-human leucocyte antigen (HLA) antibodies to specific HLA antigens, and evaluation of the psychosocial milieu of the patient and the patient's family that may affect post-transplant management. Transplant should be considered in certain anatomic and physiologic conditions with or without associated ventricular dysfunction. These conditions may include surgically uncorrectable severe stenosis or atresia in the proximal coronary arteries, severe stenosis and/or insufficiency in systemic ventricular valves, severe arterial oxygen desaturation from a cardiac cause, persistent protein-losing enteropathy and/or plastic bronchitis associated with CHD despite optimal medical-surgical therapy, and pulmonary hypertension with the potential risk of developing fixed, irreversible elevation of PVR that could preclude transplant in the future. Transplant alone should not be performed in patients with severe, irreversible disease in other organ systems or when it is part of a severe, irreversible multisystemic disease process. In such cases, multiorgan transplantation may be considered. Transplant alone should not be performed in the presence of severe hypoplasia of the central branch pulmonary arteries or pulmonary veins or as routine primary therapy for any specific congenital heart lesion before attempted or considered surgical repair.

Intraoperative monitoring and induction [36-40] Essential monitors

Standard American Society of Anesthesiologists monitors, including pulse oximetry (bilateral), noninvasive blood pressure monitor, 5-lead electrocardiography, and gas analysis should be established before induction of anesthesia. Additional monitors include invasive arterial blood pressure (ideally before induction), central venous pressure, large-bore intravenous access, PA catheter, and transesophageal echocardiogram. Femoral arterial catheter is placed in addition to a radial arterial catheter for congruence and also as a backup monitoring line during surgery. Aseptic techniques should be maintained during line placement and throughout the case to minimize the risk of line-associated infections arterial lines may be more difficult to place by palpation in patients with LVADs thus ultrasound equipment should be readily available. A central line is required for inotrope and vasopressor administration, monitoring, and for volume administration. Many anesthesiologists prefer to place a PA catheter before induction to help guide their hemodynamic management during induction. Given the significantly higher incidence of intraoperative awareness for patients undergoing cardiopulmonary bypass use of processed electroencephalogram technologies may be beneficial. Cerebral oximetry has also demonstrated utility for patients undergoing cardiac surgery however, further research in this area is needed. Placement of a PA catheter is useful to assess pulmonary artery pressures and assess right-sided cardiac output in both the operating room and the intensive care unit. However, successful positioning of the PA catheter can be difficult secondary to right ventricle (RV) dilation, tricuspid regurgitation, and frequent arrhythmias. Additionally, the utilization of PA catheter has decreased secondary to associated complications from placement, inaccuracy of derived hemodynamic values, and inconsistencies with data interpretation. It is important to note that continuous cardiac output monitoring from a PA catheter averages data from the past 5 to 15 minutes. Average in vivo time delays associated with sudden changes in CO of critically ill patients were reported to be $9.3,10.5$, and 11.8 minutes for a $50 \%, 75 \%$, and $90 \%$ response, respectively. Despite these limitations, the ability to continuously assess PA pressures is often crucial for intraoperative and postoperative management.

Additional monitors as per institutional protocol

- $\quad$ BIS guided depth anesthesia

- Transcutaneous cerebral oximetry

- Near InfraRed Spectrophotometry.

- Mixed venous oximetry

- Continuous cardiac output Monitoring.

- Continuous ABG monitoring.

\section{TEE Role in Cardiac transplant}

The use of transesophageal echocardiography (TEE) for all patients undergoing cardiac transplant has been suggested and it currently has a class II indication by the American Society of Echocardiography. TEE is invaluable in assessing ventricular function, contractility, volume status and the detection of intracardiac thrombi. TEE is used before bypass to assess biventricular function, contractility, volume status, pleural effusions, aortic atherosclerosis, and the detection of intracardiac thrombosis as well as aortic atherosclerosis in the pre-bypass period. TEE is crucial to 
facilitate separation from CPB. Particular concerns during separation include evaluation of intracardiac air, ventricular function (RV function), valvular function, and absence of intracardiac shunts. TEE guidance also allows for early detection of hemodynamic compromise and facilitates rapid therapeutic intervention to maintain hemodynamic stability

\section{Induction anaesthsia}

- Risk for cardio-respiratory collapse is high. The induction sequence is of critical importance as these patients are highly dependent on endogenous sympathetic drive and anesthetic agents, which ablates the latter combined with drug-induced reductions in heart rate inotropic function, and preload can produce sudden cardiovascular collapse so Surgeon/perfusionist must be present for urgent sternotomy and CPB initiation.

- External defibrillator pads

- Hemodynamic goals

Adequate preload

- Maintain elevated systemic vascular resistance.

- Adequate heart rate to maintain cardiac output.

- Induction results in decreased contractility, increased venous capacitance, and decreased vascular tone. Continuation of all preoperative inotropes and vasoconstrictive agents is essential. Patients with end-stage heart failure have down regulation of the beta-adrenergic receptors and will require potentially higher doses of beta-agonist inotropes.

- $\quad$ Reduced cardiovascular function entails early use of pressor and inotropes like epinephrine, norepinephrine, or dobutamine. Vasopressin increases systemic vascular resistance with less impact on pulmonary vascular resistance.

- Immediate availability of blood and blood products is essential.

- $\quad$ CMV status of the donor and of the recipient is needed to determine whether CMV-negative packed red blood cells should be ordered. Additionally, patients with ventricular assist devices should be placed on a higher risk for postbypass bleeding.

- Mechanical ventilation be initiated with a high inspiratory oxygen concentration and small inspiratory tidal volumes $(6 \mathrm{~mL} / \mathrm{kg}$ ) with optimal PEEP to maintain functional residual capacity utilizing intraoperative spirometry. Ventilatory management should be focused on its effects on pulmonary vascular resistance and right ventricle function.
- Alveolar hypoxia and hypercarbia are potent pulmonary vasoconstrictors, whereas hypocarbia is a pulmonary vasodilator.

Pre-bypass period

- Maintenance of anesthesia involves inhalational agents along with moderate doses of opioids. Antifibrinolytic therapy should be started before cardiopulmonary bypass in most cases.

- $\quad$ Specific antibiotics and immunosuppressive agents should be given before incision as per institutional protocol.

- Heparin is administered before aortic cannulation.

- Before superior vena cava cannulation, the PA catheter should be withdrawn from the heart into the superior vena cava.

- $\quad$ Cardiac transplant surgery is performed through a median sternotomy incision, using CPB in much the same way as other common cardiac surgical procedures.

- Cannulation of the aorta is often higher in the aortic arch than traditional cardiac surgery and is followed by individual cannulation of the superior and inferior vena cava.

\section{Surgical phases}

Orthotropic cardiac transplant has 3 surgical approaches;

1. Biatrial approach involves four major anastomoses of the procedure are the left and right atrial anastomoses and the end-to-end aortic and pulmonary anastomoses.

2. Bicaval method involves five areas of anastomoses, including the left atrial cuff, superior vena cava and inferior vena cava, PA, and aorta. The right atrium of the recipient is excised leaving behind a 2-3 cm cuff around each cava (cavoatrial cuff). The left atrial excision removes the left atrial appendage leaving a small cuff around the 4 pulmonary veins. The donor left atrium is sutured to the recipient left atrium before the inferior vena cava and the superior vena cava are sutured to the recipient cavoatrial cuff. The biggest advantages of this technique are lower incidence of tricuspid regurgitation and conduction disturbances.

3. Total transplantation technique involves total transplantation with 8 anastomoses sites and is rarely used.

4. Heterotopic transplantation is usually indicated in situations characterized by gross disparity in size between donor and recipient hearts and irreversible pulmonary hypertension and involves end-to-side aortic and pulmonary anastomoses and biatrial connections. 
Weaning from cardiopulmonary bypass

Protocolized review should be performed to prepare for separation from CPB. The patient should be warm ( $>35.5 \mathrm{C}$ ), have adequate level of sedation, ventilation should be initiated with verification of bilateral lung expansion, arterial blood gas should be reviewed, and electrolytes should be corrected. Identification of vascular resistance can be estimated by mean arterial pressure (MAP) on full-flow CPB and vasoconstrictive agents should be considered for MAP less than $60 \mathrm{~mm} \mathrm{Hg}$. Initial TEE evaluation should focus on retention of air in area of anastomosis as well as along the left ventricular (LV) apex. After cross clamp removal, direct-acting inotropic agents should be started, given the absence of reflexmediated heart rate responses. Standard deairing maneuvers are performed; at the author's institution routinely, methylprednisolone $1 \mathrm{~g}$ prior to cross clamp release. Following removal of the cross clamp, some electromechanical activity usually ensues. Rewarming is completed in the usual fashion and the weaning process is begun. Choice of inotrope varies from one institution to the other; some groups advocate the routine use of isoproterenol in doses ranging from 0.005 to $0.05 \mathrm{ug} / \mathrm{kg} / \mathrm{min}$ to ensure an adequate heart rate and contractility, and some others employ dobutamine 5-15 $\mathrm{ug} / \mathrm{kg} / \mathrm{min}$. Some patients may need temporary epicardial pacing to wean from bypass and into the immediate postoperative period, indeed long-term transvenous pacing is not uncommon in heart transplant recipients owing to loss of sinus node function. Right ventricular failure or dysfunction is the most common etiology of failure to wean from CPB, heralded by mean PA pressures often greater than 25-30 mmHg. Management of this situation involves maintaining adequate oxygenation and ventilation, avoiding acidosis, hypercarbia, hypothermia and the use of appropriate pulmonary vasodilators (prostaglandin E1, nitric oxide, nitroglycerine, sodium nitroprusside) and inotropes that support the right ventricle (dobutamine and milrinone), Failure to respond to these measures might indicates the need for mechanical right heart support. [18] Aggressive measures to reduce pulmonary vascular resistance are essential. Use of chronotropic agents, such as isoproterenol is more common compared with other CPB procedures. Use of atrial and ventricular pacing also is not uncommon, with $4 \%$ to $12 \%$ of patients requiring a permanent pacemaker from loss of sinus node function.41 During weaning from CPB, volume within the heart should be restored slowly and the PA catheter should be repositioned. Of particular concern for the HT recipient is the PVR and RV function. The transplanted heart should be carefully loaded with close monitoring of RV function and PA pressures given that the naive RV can acutely fail when exposed to pressures higher than 45 mm Hg.41 To reduce stress on RV function, one must minimize PVR and improve inotropy. Ventilation strategies described previously to reduce PVR should be reinitiated. Beta-adrenergic agents, such as epinephrine (0.05-0.4 $\mathrm{mg} / \mathrm{kg}$ per minute). or dobutamine (3-8 $\mathrm{mg} / \mathrm{kg}$ per minute), should be initiated to improve inotropy. Phosphodiesterase inhibitors, such as milrinone, are common medications used to decrease PVR and improve contractility, but these also before separation from bypass, a full TEE evaluation should be performed. Specific areas of interest include the following: 1. Evaluation of RV size and function 2. Evaluation of tricuspid regurgitation 3. Evaluation for turbulent flow across anastomoses sites 4. Atrial sizes 5. LV size and function 6. Mitral regurgitation 7. Presence of pericardial effusions 8. Diastolic function.

\section{Transplanted Heart physiology [41-46]}

The newly transplanted heart needs appropriate filling pressures, being completely preload dependent. However, one must be vigilant to avoid high central venous pressures and right ventricular over distension. Meticulous attention is paid to the maintenance of adequate oxygenation and ventilation, intravascular volume, pulmonary and systemic pressures, normothermia and coagulation. Appropriate antirejection and immunosuppressive regimens are instituted (typically prednisone, tacrolimus, azathioprine and cyclosporine combinations). Most patients are maintained on inotropic and chronotropic support for 36-72 hours. Extubation is typically achieved when haemodynamics is stable and bleeding is no longer a risk. Inotropic support is withdrawn gradually, and invasive monitoring is removed; chest tubes are usually taken out after 24 hours. Cardiac denervation is an inevitable consequence, as the cardiac plexus is divided in the donor, resulting in a denervated donor heart. The atrial remnant of the recipient remains innervated, but no impulses will cross the suture line. As a result, the donor atrium is responsible for heart rate generation. The transplanted heart has a higher intrinsic rate and reduced rate variability. Resting heart rates range from 90 to 110 beats per minute. Normal responses to changes in position like orthostatic changes, are lost as are the variations in response to stimuli such as the Valsalva maneuver, carotid sinus massage as well as normal responses to laryngoscopy and intubation. This has obvious implications for the anesthesiologist. Intrinsic functions such as cardiac impulse formation and conduction are intact. The Frank-Starling mechanism is also intact. In the innervated heart, the normal acute response to a sudden reduction in intravascular volume is a simultaneous increase in both heart rate and contractility. In the denervated heart, however, the initial response via the Frank-Starling mechanism is an increase in stroke volume dependent on an adequate left ventricular end diastolic volume. The increased contractility secondary to heart rate is a secondary effect and is dependent on circulating catecholamines. The transplanted heart is, therefore, critically preload dependent; higher filling pressures are needed, and this has to be kept in mind before the induction of general and 
regional anesthesia. In terms of altered drug effects, it is now well known that drugs or maneuvers that act via autonomic nerve fibres are ineffective in the transplanted heart. Vagolytic agents such as pancuronium, atropine and glycopyrrolate have no effects on heart rate and may have undesirable effects in heart transplant recipients. The response to anticholinesterases is unpredictable. The heart retains its responsiveness to direct acting agents such as isoproterenol, epinephrine, norepinephrine, dopamine and dobutamine. Other features noted in the transplanted heart include mild to moderate mitral and tricuspid regurgitation.

\section{Postoperative cardiac transplant issues [17,18,23-27]}

Often the transplanted donor heart systolic function is very good for both the LV and RV; however, this may worsen over the next 12 hours depending on several factors, such as total ischemic time, donor LV wall thickness, or acute rejection the right heart function may worsen in minutes depending on, for example, preexisting pulmonary hypertension, pulmonary edema, volume overload, or protamine reaction. Any pathologic increase in right ventricular after load can be detrimental to an unconditioned heart causing decreased right-sided cardiac output with dilation of the $\mathrm{RV}$, increasing tricuspid regurgitation, and an under filled LV.

Complications in the early postoperative period include hyperacute and acute rejection, pulmonary and systemic hypertension, cardiac arrhythmias, respiratory failure, renal failure and infection in the immunocompromised patient. Early rejection is divided into acute and hyperacute based on pathology. Acute rejection occurs within the first 6 months after transplantation. It manifests as general dysfunction from low cardiac output to arrhythmias and is caused by the recipient's immune response to the foreign tissue. Testing includes taking serial endomyocardial biopsies of the transplanted heart, invasive monitoring, and frequent echocardiography. Treatment entails more aggressive immunosuppression therapy. Hyperacute rejection occurs within minutes to hours and is rare. Preformed recipient antibodies to HLA class I on the vascular endothelium of the heart cause complement activation and fixation leading to cell death, thrombosis, ischemia, and heart failure. The only chance for survival is mechanical support until another heart can be transplanted. Primary graft failure is defined by most as single or biventricular dysfunction within the first 24 hours after transplantation manifesting as severe hemodynamic instability requiring 2 or more inotropes or mechanical support. It is the most common cause of early mortality in the first 30 days after transplantation. The etiology is thought to be from ischemia reperfusion injury with myocardial stunning. Some factors that contribute are increasing age of donor, donor heart dysfunction, increased isch- emic time, donor-recipient size mismatch, and recipient factors, such as pulmonary hypertension. Treatment is supportive with inotropic and/or mechanical agents until the heart recovers. The major limiting factor in the long-term outcome after heart transplantation has been allograft coronary artery disease to the extent that it is the major cause of death after the first year. Its incidence ranges from $10 \%-20 \%$ at 1 year and has been found to be as high as $50 \%$ by 5 years post-transplant. This transplant vasculopathy is an insidious process and has been well characterized [19]. In contrast to non-transplant related arteriosclerosis, it is usually diffuse and involves the vessel circumferentially and has no particular predilection for specific vasculature. It is considered to be a pan vasculopathy. The exact immunomechanisms of graft arteriopathy are poorly understood. Some of the factors that predispose to allograft coronary artery disease (CAD) are speculated to be older donor age, male sex, hypertension in the donor, hypertension in the recipient, African-American recipient, immunosuppression and CMV infection. Overall survival has improved over the past 40 years climbing from a 5-year survival rate of $70 \%$ in the early 1990 s to $77 \%$ in 2004. Post-transplant renal dysfunction is common and is primarily the result of cyclosporine nephrotoxicity. Hypertension is common, occurring in approximately two-thirds of all cardiac transplant recipients, attributed to the use of cyclosporine and corticosteroids both mainstays of immunosuppressive therapy. Chronic immunosuppression is associated with an increased incidence of malignant neoplastic disease. It has been estimated that transplant recipients have a 100 times greater risk for developing cancer than the general population. Cancers seen in the heart-transplant recipient include those of the skin as well as posttransplant lymphoproliferative disorders.

\section{Newer dimensions in cardiac transplant [22,47-49]}

Xenotransplantation this refers to the use of organs from other species, specifically porcine and non-human primate hearts. This offers a theoretical alternative to allograft transplantation, but is limited by multiple issues such as the immune responses to xenograft antigens as well as complex ethical and societal dilemmas. 'Cardiac Reduction:' This procedure introduced by Batista, involves reducing the size of the left ventricle by removing a portion of it, usually the lateral wall on the theoretical premise that wall tension will be reduced in a smaller chamber and that reducing the mass to diameter ratio of the heart improves pump function. Part of this procedure involves mitral valve repair, which improves the mitral regurgitation associated with severe dilated cardiomyopathy. However, much of the evidence over the past decade has indicated lack of success with this procedure. This procedure was introduced as a potentially promising therapy for patients with dilated cardio- 
myopathy and congestive heart failure, but multiple studies have shown that the reduction in left ventricular volume is insufficient to restore adequate function, clinical results have been disappointing with event free survival being poor at 2 years. Dynamic cardiomyoplasty this entails the use of the latissimus dorsi muscle that is mobilized and wrapped around both ventricles and connected to a pacing device, which causes the muscle to contract synchronously with the heart. Long-term results of this procedure have been limited by high incidences of sudden cardiac death and by the decrease in the power production of the skeletal muscle graft with time. Comparative studies have failed to demonstrate any survival improvement against medical therapy. Experimental techniques include the use of fetal myoblast cells to replace injured myocardium as well as the use of endothelial and fibroblast growth factors.

\section{Conclusions}

Heart transplantation is fraught with multiple risk and challenges faced at various levels beginning from donor management until in vivo recipient transplantation. The meticulous care and commitment of a heart transplant team can achieve what has been considered a challenge and a near impossible feat. This article simply tries to highlight the checks and precautions one needs to keep in mind while undertaking cardiac transplant.

\section{Bibliography}

1. Cooper DK. "Transplantation of the heart and both lungs. I. Historical review". Thorax 24 (1969): 383-390.

2. Reitz BA., et al. "Heart-lung transplantation: successful therapy for patients with pulmonary vascular disease". The New England Journal of Medicine 306 (1982): 557-564.

3. Yancy CW., et al. "2013 ACCF/AHA guideline for the management of heart failure: a report of the American College of Cardiology Foundation/American Heart Association Task Force on Practice Guidelines". Journal of the American College of Cardiology 62.16 (2013): e147-239.

4. Metra M., et al. "Advanced chronic heart failure: a position statement from the Study Group on Advanced Heart Failure of the Heart Failure Association of the European Society of Cardiology". European Journal of Heart Failure 9.6-7 (2007): 684-694.

5. Mandeep R. Mehra., et al. "The 2016 International Society for Heart Lung Transplantation listing criteria for heart transplantation: A 10-year update". The journal of heart and lung transplantation 35.1 (2016): 1-23.
6. Lower RR and Shumway NE. "Studies on orthotopic homotransplantation of the canine heart". Surgical forum 11 (1960): 18-19.

7. Caves PK., et al. "Diagnosis of human cardiac allograft rejection by serial cardiac biopsy". The Journal of Thoracic and Cardiovascular Surgery 66.3 (1973): 461-466.

8. DiBardino DJ. "The history and development of cardiac transplantation". Texas Heart Institute Journal 26.3 (1999): 198-205.

9. American Society of Anaesthesiology and Society of cardiovascular Anaesthesiology at Task Force on Transesophageal echocardiography. "Practice guidelines for perioperative transesophageal echocardiography. An updated report by the American Society of Anesthesiologists and the Society of Cardiovascular Anesthesiologists Task Force on Transesophageal Echocardiography. Practice guidelines for perioperative transesophageal echocardiography. An updated report by the American Society of Anesthesiologists and the Society of Cardiovascular Anesthesiologists Task Force on Transesophageal Echocardiography". Anesthesiology 112 (2010): 1084-1096.

10. Kanagarajan N., et al. "Anaesthetic management and preservation of donor heart living for transplantation". Indian Journal of Anaesthesia 48 (2004): 142-144.

11. Subramaniam K and Yared JP. "Management of pulmonary hypertension in operating rooms". Seminars in cardiothoracic and vascular anaesthesia 11 (2007): 119-136.

12. Davies RR., et al. "Standard versus bicaval techniques for orthotopic Heart transplantation an analysis of the United Network for Organ Sharing database". The Journal of Thoracic and Cardiovascular Surgery 140.3 (2010): 700-708.

13. Schnoor M., et al. "Bicaval versus standard technique in orthotopic HT: a systematic review and meta-analysis". The Journal of Thoracic and Cardiovascular Surgery 134.5 (2007): 13221331.

14. Dell'Aquila AM., et al. "Bicaval versus standard technique in orthotopic heart transplant: assessment of atrial performance at magnetic resonance and transthoracic echocardiography". Interactive CardioVascular and Thoracic Surgery 14.4 (2012): 457-462.

15. Kavarana MN., et al. "Mechanical support for the failing cardiac allograft: a single-center experience". The Journal of Heart and Lung Transplantation 22.5 (2003): 542-547. 
16. Boilson BA., et al. "Device therapy and cardiac transplantation for end-stage heart failure". Current Problems in Cardiology 35.1 (2010): 8-64.

17. Weil R 3rd., et al. "Hyperacute rejection of a transplanted human heart". Transplantation 32.1 (1981): 71-72.

18. Lindenfeld J., et al. "Drug therapy in the heart transplant recipient: part II: immunosuppressive drugs". Circulation 110.25 (2004): 3858-3565.

19. Iyer A., et al. "Primary graft failure after HT". The Journal of Heart and Lung Transplantation 2011 (2011): 175768.

20. Ramakrishna H., et al. "Adult cardiac transplantation: a review of perioperative management Part-I". Annals of Cardiac Anaesthesia 12.1 (2009): 71-78.

21. Darby JM., et al. "Approach to management of the heartbeating 'brain dead' organ donor". JAMA 261 (1989): 2222-2228.

22. Rose EA and Stevenson LW. Xenotransplantation: Management of end-stage heart disease. Philadelphia: Lippincott-Raven Publishers 1998.

23. Stein KL., et al. Intensive care of the cardiac transplant recipient. In: Ayres SM, Grenvik A, Holbrook PR, Shoemaker WC, editors. Textbook of Critical Care. 3rd ed. Philadelphia (1995): WB Saunders. p. 1649.

24. Steed DL., et al. "General surgical complications in heart and heart-lung transplantation”. Surgery 98 (1985): 739-745.

25. Rowan RA and Billingham ME. "Myocardial innervation in long-term heart transplant survivors: A quantitative ultrastructural survey". The Journal of Heart and Lung Transplantation 7.6 (1988): 448-452.

26. Uretsky BF. "Physiology of the transplanted heart". In: Thompson ME, editor. Cardiac Transplantation. Philadelphia Davis (1990): 21-56.

27. Hunt SA. "Current status of cardiac transplantation". JAMA 280 (1998): 1692-1698.

28. Goda A., et al. "The Heart Failure Survival Score outperforms the peak oxygen consumption for heart transplantation selection in the era of device therapy". The Journal of Heart and Lung Transplantation 30 (2011): 315.
29. Gorodeski EZ., et al. "Application of the Seattle Heart Failure Model in ambulatory patients presented to an advanced heart failure therapeutics committee". Circulation Heart Failure 3 (2010): 706-714.

30. Kalogeropoulos AP., et al. "Utility of the Seattle Heart Failure Model in patients with advanced heart failure". Journal of the American College of Cardiology 53 (2009): 334-342.

31. Goda A., et al. "Selecting patients for heart transplantation: comparison of the Heart Failure Survival Score (HFSS) and the Seattle heart failure model (SHFM)". The Journal of Heart and Lung Transplantation 30 (2011): 1236-1243.

32. Grossi PA. "Update in HIV infection in organ transplantation". Current Opinion in Organ Transplantation 17 (2012): 586-593.

33. Sims DB., et al. "Human immunodeficiency virus infection and left ventricular assist devices: a case series". The Journal of Heart and Lung Transplantation 30 (2011): 1060.

34. Castel MA., et al. "Heart transplantation in HIV infected patients: more cases in Europe". The Journal of Heart and Lung Transplantation 30 (2011): 1418.

35. Goldberg SW., et al. "Adults with congenital heart disease and heart transplantation: optimizing outcomes". The Journal of Heart and Lung Transplantation 33 (2014): 873-877.

36. Murkin JM., et al. "Monitoring brain oxygen saturation during coronary bypass surgery: a randomized, prospective study". Anesthesia and Analgesia 104.1 (2007): 51-58.

37. Deschamps A., et al. "Cerebral oximetry monitoring to maintain normal cerebral oxygen saturation during high-risk cardiac surgery: a randomized controlled feasibility trial". Anesthesiology 124.4 (2016): 826-836.

38. Marik PE. "Obituary: pulmonary artery catheter 1970 to 2013". Annals of Intensive Care 3.1 (2013): 38.

39. Leibowitz AB and Oropello JM. "The pulmonary artery catheter in anesthesia practice in 2007: an historical overview with emphasis on the past 6 years". Seminars in Cardiothoracic and Vascular Anesthesia 11.3 (2007): 162-176.

40. Shanewise J. "Cardiac transplantation". Anesthesiology Clinics of North America 22.4 (2004): 753-765.

41. Holmes CL., et al. "Science review: vasopressin and the cardiovascular system part 2-clinical physiology". Critical Care 8.1 (2004): 15-23. 
42. John R. "Donor management and selection for HT". Seminars in Thoracic and Cardiovascular Surgery 16.4 (2004): 364-369.

43. Khush KK., et al. "Donor cardiac troponin I levels do not predict recipient survival after cardiac transplantation". The Journal of Heart and Lung Transplantation 26(10) (2007): 1048-1053.

44. Kuppahally SS., et al. "Outcome in cardiac recipients of donor hearts with increased left ventricular wall thickness". American Journal of Transplantation 7.10 (2007): 2388-2395.

45. Welp H., et al. "Sex mismatch in HT is associated with increased number of severe rejection episodes and shorter long-term survival". Transplantation Proceedings 41.6 (2009): 2579-2584

46. Ratcliffe M. "Batista's operation: What have we learned?". Journal of the American College of Cardiology 36 (2000): 21152118.

47. 47. Starling RC and McCarthy PM. "Partial left ventriculectomy: Sunrise or sunset?". European Journal of Heart Failure 1 (1999): 313-317.

48. Acker MA. "Dynamic cardiomyoplasty: At the crossroads". The Annals of Thoracic Surgery 68 (1999): 750-755.

Volume 3 Issue 12 December 2019

(c) All rights are reserved by Sandeep Kumar Kar and

Pallav Mishra.

Citation: Sandeep Kumar Kar and Pallav Mishra. "An Overview of Anesthesia Practices for Heart Transplant in India”. Acta Scientific Pharmaceutical Sciences 3.12 (2019): . 